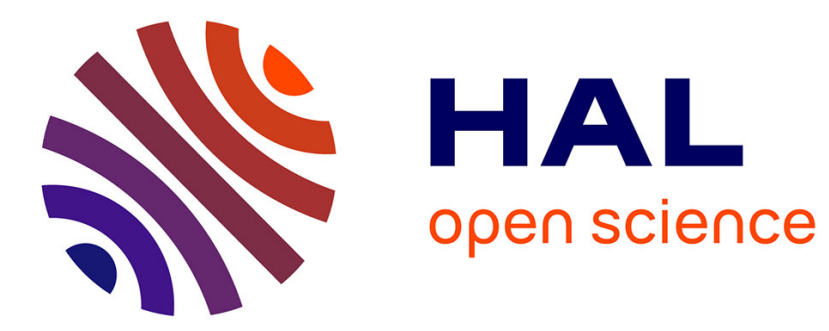

\title{
ÉTUDE DES CHAMPIGNONS TROUVÉS DANS LE LAIT LA CRĖME ET LE BEURRE (Suite)
}

\author{
M. Grimes, H. A. Cummins, V. C. E. Kennelly
}

\section{To cite this version:}

M. Grimes, H. A. Cummins, V. C. E. Kennelly. ÉTUDE DES CHAMPIGNONS TROUVÉS DANS

LE LAIT LA CRÈME ET LE BEURRE (Suite). Le Lait, 1932, 12 (120), pp.1071-1079. hal-00895086

\section{HAL Id: hal-00895086 \\ https://hal.science/hal-00895086}

Submitted on 1 Jan 1932

HAL is a multi-disciplinary open access archive for the deposit and dissemination of scientific research documents, whether they are published or not. The documents may come from teaching and research institutions in France or abroad, or from public or private research centers.
L'archive ouverte pluridisciplinaire HAL, est destinée au dépôt et à la diffusion de documents scientifiques de niveau recherche, publiés ou non, émanant des établissements d'enseignement et de recherche français ou étrangers, des laboratoires publics ou privés. 
La suite de notre étude se propose précisément de répondre à ces diverses questions, en vue d'arriver à fixer une méthode analytique sérieuse fondée sur des bases sûres.

\title{
BIBLIOGRAPHIE.
}

[1] M. H. Mac Crady. Tables for rapid interpretation of fermentation tube results. Publ. Health Journ. Toronto, 1918, IX, pp. 201 à 220. Cité dans : Karl J. Demeter. Bakteriologische und biologische Untersuchungsmethoden. Handbuch der Milchwirtschaft, 1930, p. 372.

[2] W. Dorner et P. Demont. Recherches sur le procédé Burri de numération bactérienne. Le Lait, décembre 1931, pp. 1015-1016.

(A suivre.)

\section{ÉTUDE DES CHAMPIGNONS TROUVÉS DANS LE LAIT LA CRËIME ET LE BEURRE}

\author{
par \\ M. GRIMES \\ Section de Bactériologie laitière \\ et \\ H. A. CUMMINS et V. C. E. KENNELLY \\ Sertion de Botanique \\ Université de Cork (Etat libre d'Irlande). \\ (Suite).
}

Mucor racemosus Fres. (fig. 7, Pl. 17).

Mycelium rampant et dense, couleur variant du jaune sale au blane ou gris. Sporanges terminaux, sur sporangiophores ramifiés ou non ramifiés, de dimensions variables, et jaunes brunâtres. Les sporangiophores sont de longueur variable. Les chlamydospores sont caractéristiques, et présentes à la fois dans le mycelium et dans les sporangiophores. Elles sont globulaires, ou en forme de casque et contiennent des spores brillantes. La columelle est en forme de massue. La capsule se brise quand on la monte dans l'acide lactique et le bleu coton pour l'examiner et laisse un col autour de la columelle.

Fait cailler le lait-tournesol d'une façon très épaisse en 48 heures, changeant le bleu en blane pur, avant traces de couleur rouge à la surface.

Cultures sur lait. - Lait caillé en 48 heures, avec décoloration du tournesol, excepté à la surface, suivi par solubilisation lente du caillé.

Tolérance à la chaleur. - Croissance à $70^{\circ} \mathrm{C}$. Pas de croissance à $75^{\circ} \mathrm{C}$.

Tolérance au sel. - Pas de croissance en solution de CzapeK contenant $15 \%$ de NaCl.

$p H$. - Sur solution de CzapeK, le $p H$ du milieu devint 6,8 .

Gélatine. - Liquéfaction en 10 jours, coloration verte sur la surface inférieure.

Production de diastase. - Faible.

Mucor corymbifer Cohn (Absidia Lichtheimi Ledner) (fig. 8, Pl. 17).

Isolé à trois occasions. Il a un mycelium gris blanchâtre tressé lâchement, se 
développant sur les autres cultures. Les hyphes sont longs, se courbant vers le bas en venant en contact avec le couverele de la boîte de PETRI.

Les sporanges, quoique très petits, sont visibles à l'œil nu comme de petites taches sombres. Les sporangiophores portent un sporange terminal et des sporangiophores latéraux quelquefois en forme d'ombelle.

Les plus grands sporanges mesurent $70 \mu$ de diamètre, les plus petits 10-20 $\mu$. Ils sont brun sombre, et des apophyses existent qui sont d'abord légèrement colorées, puis deviennent sombres avec l'âge. Les arrangements de la fig. 8, A, sont nombreux dans le milieu. Ils sont irréguliers de forme et de couleur brun verdâtre avec contenus granuleux. Des projections du corps prineipal de la masse se développent en mycelium sans couleur. Nous ne pouvons pas trouver de référence pour ces corps dans la documentation que nous possédons ; de pareils corps sont apparus dans le M. Mucedo (fig. 10, B et C). Dans les cultures, le lait-tournesol est coagulé avec production d'acide, caillé mou et séparation de sérum.

Cultures sur lait. - Coagulation avec production d'un caillé acide et mou et séparation de sérum.

Tolérance à la chaleur. - Croissance à $70^{\circ} \mathrm{C}$. Pas de croisrance à $75^{\circ} \mathrm{C}$. Cette moisissure se développait bien quand elle était maintenue à $5^{\circ} \mathrm{C}$.

Tolérance au sel. - Croissance en 2 semaines en solution de Czaper contenant $10 \%$ de $\mathrm{NaCl}$. Chlamydospores formées en solution de Czaper contenant $3 \%$ et $5 \%$ de $\mathrm{NaCl}$.

$p H$. - Peu ou pas de changement dans la réaction de la solution de CzapeK avec et sans addition de $\mathrm{NaCl}$.

Gélatine. - Liquéfaetion partielle en 7 jours. Le mycelium se développant dans la partie liquide ressemblait à l'Oädium, avec coloration verte sur la surface inférieure.

Production de diastase, - Faible.

Mucor mucedo Linne (fig. 10, Pl. 17).

Forme très commune avec mycelium blane sale et têtes noires. Le mycelium ramifié dans le milieu alimentaire donne naissance à des sporangiophores dirigés en haut, non ramifiés. Sporanges $=70-140 \times 50-80 \mu$. Spores $=6-12 \times 3-6 \mu$ Des arrangements analogues à ceux du Mucor corymbifer (fig. 8, A, 10, B et C) furent trouvés, qui, transportés sur milieu frais, donnèrent des tubes, comme dans le $M$. corymbifer, se développant en mycelium ordinaire. Ces chlamydosspores (?) apparurent à nouveau au fur et à mesure que la culture vieillissait.

Mucor alternans Van Tieghem (fig. 9, Pl. 17).

Croissance jaune sale basse et rampante, n'ayant pas l'apparence microscopique habituelle d'un Mucor. Des sporanges de diverses dimensions sur de courtes tiges, se ramifiant comme sur le dessin. Spores $=2-3 \times 5-7 \mu$. Cultivé sur laittournesol, le caillé est coagulé et le tournesol décoloré. Il y a une forte croissance du champignon sur la surface avee hyphe aérien de $1-1 \frac{1}{4}$ pouce ' $(2 \mathrm{~cm} .5$ à $3 \mathrm{~cm} .1$ ) de haut.

Thamnidium elegans Link (fig. 11, P1. 17).

Considéré entièrement comme saprophyte. Quand on le cultiva pour la première fois, il se développa rapidement, et il infecta une boîte, ouverte pour exa- 
men, avec une très forte croissance. Les formes jeunes sont difficiles à distinguer d'un Mucor jusqu'à ce que les sporanges secondaires apparaissent. Spores $=8-10 \times 6-8 \mu$; mêmes dimensions pour les sporanges primaires et secondaires. Les sporanges secondaires tombent généralement intacts, et, en cultures sur solution de sucre à $2 \%$, atteignaient 7 ou 8 fois leurs dimensions originelles sans montrer de signes d'éelatement. Sur lait-tournesol, la coagulation du caillé a lieu avec décoloration du tournesol. Le caillé est mou, avec séparation marquée du sérum et voile à la surface.

Sporotrichum carnis Brooks et Hansford [4] (fig. 3, Pl. 17 ; fig. 1, A et B, P1. 18).

Tous les spécimens trouvés avaient la forme caractéristique de deux ou trois cercles concentriques, "couverts d'une croute ", ou d'apparence cristalline, avec de petites gouttelettes (fig. 1, A, Pl. 18) variant de $3 \frac{\mathrm{m}}{\mathrm{m}} 12$ à $3 \frac{\mathrm{m}}{\mathrm{m}} 9 \mathrm{de}$ diamètre.

Sur lactose-agar nutritif, il y avait une bonne croissance blanche semblable à du feutre, la couleur tournant au jaune crémeux, avec gouttelettes jaune brillant sur les petits morceaux les plus vieux. Ces petits morceaux entourés et joints par le mycelium ne portent pas de branches aériennes. Centres des petits morceaux soulevés et sous-surface jaune s'obscurcissant en brun orange et concave.

Sur lait-tournesol, après 14 jours, il y avait une croissance mince, genre papier et la couleur du tournesol n'était pas changée; coloration verte sur le dessous du champignon.

Après 27 jours, le tournesol se colora en ambre, une coloration légèrement pourpre restant au sommet.

Cultures sur lait. - Croissance mince, genre papier, coagulation lente suivie par solubilisation.

Tolérance à la chaleur. - Pas de croissance après chauffage à $60^{\circ} \mathrm{C}$. Après séjour à $5^{\circ} \mathrm{C}$. pendant 3 semaines, assez bonne croissance, couleur blane pur.

Tolérance au sel. - Bonne croissance pendant 2 semaines en solution de Czaper contenant $5 \%$ de $\mathrm{NaCl}$, légère en solution de Czaper contenant 10\% de $\mathrm{NaCl}$, et pas de croissance en solution de Czaper contenant $15 \%$ de $\mathrm{NaCl}$.

$p$ H. - Peu ou pas de changement dans la réaction de la solution de CzapeK avec ou sans addition de $\mathrm{NaCl}$.

Gélatine. - Gélatine liquéfiée de $50 \%$ en 10 jours.

Production de diastase. - Faible.

Trichoderma lignorum Tode $([] 5]$, vol. 8, p. 110) (Pl. 22, fig. 8, A, B et C).

Mycelium recouvert de petites touffes ressemblant à de la laine, grumeleux, rampant, d'abord blanc puis passant au vert par le jaune. Vieilles cultures saupoudrées de mycelium blane stérile. Conidies arrondies, lisses, vertes, mesurant 2,5-3 $\mu$ de diamètre, et portées en têtes aux extrémités de courts conidiophores (Pl. 22, fig. 8, A). Des chlamydospores d'environ $10 \mu$ de diamètre (fig. $8, \mathrm{~B}$ et C) furent trouvé $\in \mathrm{s}$ en nombre énorme dans des cultures sur lactose incliné maintenues à une température inférieure à $8^{\circ} \mathrm{C}$. Elles furent aussi observées dans les cultures inclinées maintenues à $21^{\circ} \mathrm{C}$.; mais, dans ce dernier cas, elles étaient rares. Ces chlamydospores avaient des parois hyalines épaisses, contenaient des corps réfringents, et étaient ou terminaux ou intercalaires sur l'hyphe. Horne et WiLliamson 
décrivent des corps analogues comme des "macrospores " dans leur Eidamia viridiscens, que Tном considère être une espèce de Trichoderma (Ref. [2],p. 542).

Cultures sur lait. - Solubilisation partielle avec production d'acide en 5 jours.

Tolérance à la chaleur. - Croissance à $80^{\circ} \mathrm{C}$. Pas de croissance à $85^{\circ} \mathrm{C}$. Maintenu à une température inférieure à $8^{\circ} \mathrm{C}$., bonne croissance de mycelium stérile, mais, à une température inférieure à $5^{\circ} \mathrm{C}$., pas de eroissance apparente.

Tolérance au sel. - Croissance faible en solution de Czapek contenant $12 \%$ de $\mathrm{NaCl}$. Pas de croissance en solution de CzapeK contenant $15 \%$ de $\mathrm{NaCl}$.

$p H$. - Réaction du milieu inchangée dans culture en solution de CzapeK avec et sans addition de $\mathrm{NaCl}$.

Gélatine. 一 Liquéfaction partielle - $1 / 4$ à $1 / 3$ - en 10 jours.

Production de diastase. - Bonne.

Cette moisissure fut aussi trouvée croissant sur des parties de planches importées pour être employées à la construction.

Acrostalagmus cinnabarinus Corda (Verticillium cinnabarinum Reink et

Berthold) (fig. 2, Pl. 17).

Apparaît fréquemment dans les cultures sous forme d'un mycelium blane touffu, devenant ultérieurement rouge brique sur tous les milieux. Le champignon s'étend, couvrant les autres végétations sans les abîmer. Le mycelium stérile ressemble beaucoup au mycelium du Phoma avant l'apparition de fructifications ayant une apparence contournée plutôt fanée. Les conidies sont maintenues ensemble par du mucus, formant une tête qui, quand elle est jeune, a l'apparence blanche brillante de gouttelettes d'eau, mais devient rouge brunâtre à maturité. La fig. 2 A montre l'apparence à un faible grossissement. La fig. $2 \mathrm{~B}$ montre les têtes conidiales à un fort grossissement. Croît faiblement sur piqûre dans la betterave, faiblement aussi sur carotte.

Cultures sur lait. - Réaction alealine et solubilisation.

Tolérance à la chaleur. - Croissance à $70^{\circ} \mathrm{C}$., sous forme d'un voile blane submergé. Pas de croissance à $75^{\circ} \mathrm{C}$. Pas de croissance apparente quand il est maintenu 3 semaines aे $5^{\circ} \mathrm{C}$.

Tolérance au sel, - Pas de croissance en solution de Czaper contenant $15 \%$ de $\mathrm{NaCl}$.

$p$ H. - Légère modification ou pas de modification de la réaction dans les eultures en solution de CzAPEK avec et sans addition de NaCl.

Gélatine. - Liquéfaction en 10 jours, coloration rouge brique dans le milieu. Production de diastase. - Bonne.

Botrytis cinerea Pers. (fig. 12, P1. 17 ; fig. 3, Pl. 18).

Est apparu à trois occasions sous forme de touffes légèrement brun grisâtre. En eultures, a produit un sclérote et des microconidies sur le mycelium, comme dans la fig. 12, PI. 17. Les hyphes sont vert sombre, et les conidies, d'abord sans couleur, sont ultérieurement teintées de brun. Des hyphes stériles turent produits aux extrémités des myceliums. Se développa bien aussi sur sections de pommes de terre, produisant un résultat similaire. Spores 9-12 $\times 6-10 \mu$, deux sortes de conidies. Sur lait-tournesol, croissance en surface et production d'une mauvaise odeur.

Cultures sur lait. - Acide, coagulation suivie par solubilisation. 
Tolérance à la chaleur. - Croissance à $65^{\circ} \mathrm{C}$. Pas de croissance à $70^{\circ} \mathrm{C}$. Conservé à $5^{\circ} \mathrm{C}$., croissance normale, mais avec mycelium duveteux stérile.

Tolérance au sel. - Peu ou pas de croissance pendant 2 semaines en solution de Czapek contenant $10 \%$ de $\mathrm{NaCl}$.

$p \mathrm{H}$. - $p \mathrm{H}$ du milieu amené à 6 `ur solution de CzAPEK.

Gélatine, - Liquéfaction partielle en 10 jours.

Production de diastase. - Suffisante.

Trichothecium roseum Link (fig. 5, Pl. 17 ; fig. 2, A et B, P1. 18).

Apparaît assez fréquemment et forme une couche poudreuse variant en épaisseur suivant le milieu; d'abord blanc, devenant ultérieurement rose pâle. Le mycelium est étalé, ramifié et cloisonné.

Les hyphes sont blanes, les conidiophores verticaux, rarement cloisonnés, le plus souvent non ramifiés, et légèrement renflés à leur extrémité. Les conidies sont terminales, formées l'une après l'autre, mais elles restent suspendues quand elles sont entièrement développées, formant une tête. Elles sont en forme de poire, bicellulaires, et très légèrement contractées à la paroi de séparation. Elles sont d'abord hyalines, puis rose pâle.

Sur lait-tournesol, la production de spores est très dense, formant une couche ferme à la surface, avec la coloration rose caractéristique. La solübilisation du lait a lieu avec réaction légèrement acide, et avec un dépôt légèrement coloré au fond du tube.

Tolérance à la chaleur, - Croissance à $65^{\circ} \mathrm{C}$. Pas de croissance après avoir été chauffé à $70^{\circ} \mathrm{C}$.

Tolérance au sel. - Bonne croissance en solution de Czapek contenant $5 \%$ de $\mathrm{NaCl}$. Légère croissance en solution de CzAper contenant $10 \%$ de $\mathrm{NaCl}$ et $12 \%$ de $\mathrm{NaCl}$. Peu ou pas de croissance en solution de CzaPEK contenant $15 \%$ de $\mathrm{NaCl}$.

$p \mathrm{H}$. $-p \mathrm{H}$ non modifié par culture pendant 2 semaines en solution de CzAPEK avec et sans addition de $\mathrm{NaCl}$.

Gélatine. - La gélatine est liquéfiée de $50 \%$ en 10 jours.

Production de diastase. - Suffisante.

Oospora Oidium lactis Fres. (fig. 1, Pl. 17).

En cultures sur lait, apparaît sous forme d'une production crémeuse, blanche, circulaire et plate, avec zones concentriques. Pas d'hyphe aérien, ou quelques hyphes très courts monoliformes sont dressés. Le mycelium est d'abord cloisonné et continu, se brisant ensuite en cellules individuelles. Les vieilles cultures ressemblent sur les bords au Torula.

Croît sur navet, panais et betterave, à l'obscurité. Le légume a d'abord une apparence sèche, ridée; ultérieurement, un mycelium blanc apparaît, et finalement a lieu une détérioration rapide, avec décortication des tissus et développement de gaz sentant mauvais. Exposé à la lumière, le mycelium blanc disparaît, et le champignon prend l'aspect d'un Torula, les légumes devenant une masse visqueuse.

Cultures sur lait. - Coagulation molle. Les cultures examinées produisent d'abord une légère augmentation de l'acidité du lait, mais, quand les cultures furent maintenues de 6 à 10 semaines, l'acidité du lait fut réduite de 0,18 à $0,12 \%$ d'acide lactique.

Tolérance à la chaleur. - Pas de croissance à $60^{\circ} \mathrm{C}$. 
Tolérance au sel. - Croissance en solution de Czaperk contenant $5 \%$ de NaCl. Légère croissance en solution de CzAPEK contenant $10 \%$ de $\mathrm{NaCl}$. Pas de croissa nee en solution de CzAPEK contenant $15 \%$ de NaCl.

Gelatine. - Si on se base sur la liquéfaction de la gélatine, il y a deux espèces, une liquéfiant la gélatine lentement, et une autre espèce qui ne liquéfie pas la gélatine en 8 semaines.

Production de diastase. - Négative.

\section{Sporendonema casei Desmaz.}

Coloration pendant la croissance du rouge orange au brun, suivant l'âge. Mycelium rampant, non eloisonné, avec touffes occasionnelles à branches dressées. Les spores produisant l'hyphe ont un pigment rougeâtre déposé dans leurs parois. Sporesthyalines, presque rondes, endogènes, formées en une rangée simple; parois de l'hyphe sporogène plutôt persistantes.

Cultures sur lait. - Légère coagulation à la surface du lait, croissanee blane sale à brun jaunâtre sur les parois latérales du tube à essai.

Tolérance au sel. - Bonne croissance en solution de CzAPEK contenant jusqu'à $10 \%$ de NaCl. Faible eroissance en surface en solution de CzAPEK contenant $15 \%$ de NaCl. Pas de croissance en solution de Czaper eontenant $20 \%$ de $\mathrm{NaCl}$.

Gélatine. - $50 \%$ de liquéfaction en 2 semaines.

Macrosporium sarcinula Berk (fig. 14, A, B et C, Pl. 17).

Trouvé une seule fois. Les hyphes sont d'abord légèrement colorés, puis tournent au noir brunâtre, sont cloisonnés et étalés. Les conidiophores sont très courts et enflés (fig. 15, A). Les conidies sont resserrées en chaînes, ce qui est rare sous cette forme, hyalines et lisses, jeunes, sombres et verruqueuses lorsqu'elles sont vieilles. Elles ont l'aspeet de réseau en raison de la formation de cloisons, et sont resserrées aux parois de séparation. Les spores sont très variables comme dimensions.

Stemphyllium spp. (5, vol.9, p. 215) (Pl. 22, fig. 12, A et B).

Stemphyllium botryosum Wallr. (fig. 15 A, B et C, P1. 17).

Trouvés à deux occasions. Très similaires à Macrosporium sarcinula, mais les conidies sont simples, les conidiophores plus longs et plus minces, et peuvent être ramifiés ou non ramifiés. Les conidies ne sont pas si resserrées que dans le Macrosporium sarcinula, et sont plus verruqueuses.

Une autre forme ressemble à $S$. ericoctonum $\mathrm{A}$. Br. et de Bary, mais les spores sont plus longues. Les hyphes sont très articulés et les spores $(22-38 \mu \times 8-13 \mu)$, lisses et de couleur vert brunâtre.

Cultures sur lait. - Réaction alcaline et solubilisation du caillé.

Tolérance à la chaleur. - Pas de croissance à $70^{\circ} \mathrm{C}$. Quand il est conservé à $8^{\circ} \mathrm{C}$, bonne croissance du mycelium stérile. Pas de croissance à $5^{\circ} \mathrm{C}$.

Tolérance au sel. - Croissance en solution de Czapek contenant $10 \%$ de $\mathrm{NaCl}$. Légère croissance en solution de Czaper contenant $15 \%$ de $\mathrm{NaCl}$. Pas de croissance en solution de CzAper contenant $20 \%$ de $\mathrm{NaCl}$.

$p \mathrm{H} .-p \mathrm{H}=6,6$ après croissance pendant 2 semaines en solution de CzÁPEK.

Gélatine. - Liquéfiée au $1 / 3$ en 10 jours.

Production de diastase. - Négative ou légèrement faible.

Stemphyllium ericoctonum var. (Pl. 23, fig. 4).

On a aussi trouvé une variété de cette espèce dans laquelle les spores sont 
portées en courtes chaînes, et sont plus sombres et de dimensions légèrement plus faibles, $21-36 \times 7-9 \mu$. On suppose qu'il s'agit d'une famille de $S$. ericoctonum. Cultures sur lait. - Réaction alcaline et solubilisation du caillé.

Tolérance à la chaleur. - Croissance à $65^{\circ} \mathrm{C}$. Pas de croissance à $70^{\circ} \mathrm{C}$. Pas de croissance quand il est maintenu à $5^{\circ} \mathrm{C}$. pendant 3 semaines.

Tolérance au sel. - Légère croissance en solution de Czaperk contenant $15 \%$ de $\mathrm{NaCl}$. Pas de croissance en solution de CzapeK contenant $20 \%$ de $\mathrm{NaCl}$.

$p \mathrm{H} .-p \mathrm{H}=6,6$ quand il est cultivé en solution de CzAPEK pendant 2 semaines. Gélatine. - Liquéfaction au $1 / \mathrm{s}$ en 10 jours.

Production de diastase. - Négative.

Stysanus microsporus Sace. (fig. 6, Pl, 17).

Apparu à deux occasions. Les têtes croissent seulement en groupes, sauf quand il est cultivé sur dextrose-agar. Dans ce cas, on a observé des ramifications paniculées, sans ordre, comme dans la fig. $6 \mathrm{~B}, \mathrm{Pl}$. 18. Jeune, les spores sont noires, mais avee l'âge, deviennent gris argent brillant, Hauteur de la culture sur agaz Czapek $=1 \mathrm{~m} / \mathrm{m} ;$ sur Lemco $5 \%=0 \mathrm{~m} / \mathrm{m}$. Sur lait-tournesol, une croissance très fortement convolutée est observée. Pas de production de tễtes conidiales, Donne une réaction acide.

Tolérance à la chaleur. - Pas de eroissance à $70^{\circ} \mathrm{C}$. Pas de croissance quand il est conservé à $5^{\circ} \mathrm{C}$. pendant 3 semaines.

Tolérance au sel. - Croissance en solution de CzAPEK contenant $10 \%$ de $\mathrm{NaCl}$. Peu ou pas de croissance en solution de Czapex contenant $15 \%$ de $\mathrm{NaCl}$.

$p H$. - Cultivé en solution de CzapeK, le $p H$ du milieu est modífié à 6,6 .

Gélatine. - Liquéfaction partielle en 10 jours.

Production de diastase. - Faible.

Gladosporium spp. (Syn. Hormodendrum).

Nous estimons avec Brooks [4] que la distinction entre le Cladosporium et l'Hormodendrum est une question de quantité et de caractère du bourgeonnement des premières conidies formées, et que les deux formes s'absorbent graduellement l'une dans l'autre. En raison de la difficulté de l'identification, puisque la nature de la croissance varie avec le milieu employé pour la culture, nous n'avons pas été capables d'identifier les quelques différentes espèces rencontrées pendant la durée du présent travail, mais les avons divisées en deux groupes principaux : $a$ ) type Cladosporium, avec cellules plus grandes et conidiophores pas si compliqués que le type; b) Hormodendrum, dont les conidiophores sont plus ramifiés et les cellules plus petites.

Cultures sur lait. - Croissance épaisse caractéristique, solubilisation partielle avec production d'acide.

Tolérance à la chaleur. - Pas de eroissance à $60^{\circ} \mathrm{C}$.

Tolérance au sel. - Belle croissance en solution de CZAFEK contenant $10 \%$ et $12 \%$ de $\mathrm{NaCl}$. Légère croissance en solution de Czaper contenant $15 \%$ de $\mathrm{NaCl}$. Pas de eroissance en solution de Czaper contenant $20 \%$ de $\mathrm{NaCl}$.

$p$ H. - Peu ou pas de changement dans le $p H$ en solution de Czaper avec ou sans addition de $\mathrm{NaCl}$.

Gélatine. - Gélatine liquéfiée de $50 \%$ en 10 jours.

Production de diastase. - Suffisante.

Une espèce trouvée montrait une tendance à se développer dans l'eau, formant 
un mycelium ressemblant extérieurement au Fumago. L'examen a montré la présence de spores ressemblant à celles du Cladosporium avec un peu de mycelium.

Fusarium Famille A (P1. 22, fig. 11, A, D, E et F).

Obtenu sur un échantillon de beurre. Spores en forme de faucille très aiguës à chaque extrémité jusqu'à 3 cloisons $(22-35 \times 3,5-4,5 \mu)$; quelquefois avec microconidies continues. Le Dr AsHbx (du Département impérial de Mycologie) indique que cette forme produit sur l'agar-pulpe de pomme de terre et sur l'agarQuaker oats, un plectenchyme carmin, des sclérotes bleu sombre et des sporodochies crémeuses; tandis que, sur le riz, il donne une eulture carmin dessus et jaune brun dessous, avec sclérotes; les chlamydospores sont absentes ou très courtes. Il semble intermédiaire entre les sections Discolor Wr. et Lateritium Wr., les conidies étant analogues à celles du F. salicis Fuck, mais cette espèce n'a pas encore été observée avee un plectenchyme carmin.

Cultures sur lait. - Acide, coagulation suivie par solubilisation.

Tolérance à la chaleur. - Pas de croissance à $70^{\circ} \mathrm{C}$. Cette moisissure s'est développée même à $5^{\circ} \mathrm{C}$.

Tolérance au sel. - Croissance en solution de Czaper avec addition de $12 \%$ de $\mathrm{NaCl}$. Pas de croissance en solution de Czaper contenant $20 \%$ de $\mathrm{NaCl}$.

$p H$. - Peu ou pas de changement en solution de CzApeK avec ou san addition de sel.

Gélatine. - Liquéfaction partielle en 10 jours.

Production de diastase. - Faible.

Cette espèce fut isolée d'un échantillon de beurre sur lequel s'étaient développées des plaques rougeâtres à la surface.

Fusarium, Famille B (P1. 22, fig. 11, C).

Stade microconidial, qui produisit toujours des microconidies $6 \times 3 \mu$ sur tous les milieux. Très peu de ma.croconidies; elles avaient trois cloisonnements, et très pointues aux extrémités. Sur gélatine, la surface inférieure de la colonie montra une couleur rose, sur agar Czapek un rose plus orangé.

Cultures sur lait. — Lait-tournesol décoloré.

Tolérance à la chaleur. - Croissance après chauffage à $65^{\circ} \mathrm{C}$. Légère croissance après chauffage à $70^{\circ} \mathrm{C}$. Pas de croissance après chauffage à $75^{\circ} \mathrm{C}$. Très légère croissance quand il est conservé à $5^{\circ} \mathrm{C}$.

Tolérance au sel. - En solution de CzapeK contenant $3 \%$ de $\mathrm{NaCl}$, le mycelium se rompt en spores arrondies, belle croissance en solution de CzAPEK contenant $12 \%$ de $\mathrm{NaCl}$, très légère en solution à $15 \%$ de $\mathrm{NaCl}$. Pas de croíssance avec $20 \%$ de NaCl.

$p$ H. - Peu ou pas de changement quand il est cultivé en solution de CzapeK avec et sans addition de $\mathrm{NaCl}$.

Gélatine. - Liquéfaction aux $3 / 4$ en 10 jours.

Production de diastase. - Faible.

Une eulture de cette espèce fut envoyée à Mr. S. F. AsHry, du Département impérial de Mycologie, qui déclara que le $\mathrm{D}^{\mathrm{r}} \mathrm{H}$. W. Wollenweber l'avait déterminée comme étant le F. reticulatum Mont. (Ann. Sc. Nat., $2^{e}$ Sér., 20, 379, 1843 ; Fusaria autographiæ delineata de Wollenweber, 119-121 et 938, 939). Cette espèce est aussi notée dans le Sacc. syll. tome X 726 comme étant le F. ampelodesmi Faut. 
et Roum. On la note aussi en Allemagne sur le Beta vulgaris et au Danemark sur Cucumis melo.

Fusarium, Famille C (Pl. 22, fig. 11, B).

Outre les précédentes, on obtint un Fusarium du lait. Les macrospores étaient plus en forme de saucisse que celles de l'espèce de Fusarium trouvée dans le beurre : 5 cloisonnements, et non aiguës aux extrémités; les spores étaient plus grandes que la forme sur beurre, ayant $30 \times 5-6,6 \mu$ de longueur.

Cultures sur lait. - Croissance duveteuse blanche très haute avec production d'acide, coagulation suivie par solubilisation du caillé.

Tolérance à la chaleur. - Pas de croissance à $70^{\circ} \mathrm{C}$. Pas de croissance apparente de la colonie quand il est conservé pendant 3 semaines à $5^{\circ} \mathrm{C}$.

Tolérance au sel. - Belle croissance en solution de CzAPEK contenant $12 \%$ de $\mathrm{NaCl}$. Très légère croissance en solution de Czaper contenant $15 \%$ de $\mathrm{NaCl}$. Pas de croissance en solution de Czaper contenant $20 \%$ de $\mathrm{NaCl}$.

$p H$. - Peu ou pas de changement en solution de CzAper avec et sans addition de sel.

Gélatine. - Liquéfaction aux 7/8 en 10 jours.

Production de diastase. - Faible.

(A suivre.)

\section{REVUE}

\section{LA MOUSSE DU LAIT}

\section{par M. G. GÉNIN}

Ingénieur chimiste E. P. C.

La question de la mousse du lait présente une importance de tout premier ordre dans l'industrie du lait et de ses dérivés. Dans certains cas, l'apparition de grandes quantités de mousse et la persistance de celle-ci peuvent être des causes de graves ennuis dans la fabrication, soit parce que cette mousse peut conduire à des pertes sensibles, soit parce qu'elle abrite des colonies bactériennes qui ne sont pas détruites par la pasteurisation. Dans d'autres cas, la mousse, au contraire, joue un rôle important, par exemple dans le cas de la fabrication de la crème fouettée ou de la crème glacée. De même, pour préparer facilement le beurre, on a intérêt à travailler sur une crème moussant facilement. Nous avons emprunté à l'ouvrage de O. RAHN et P. F. SHARP intitulé Physikder Milchwirtschaft, les points principaux qui nous ont permis d'établir le résumé qui suit de cette question.

LOI dE GIBBs ET thomson. - Il est nécessaire, lorsque l'on veut étudier la formation des mousses, de connaître les lois qui régissent cette formation et peut-être la plus importante d'entre elles, la loi de Gibbs et Thomson. 